\title{
A Design and Performance Evaluation of 2-Level Call Admission Control Scheme in Mobile Multimedia Networks*
}

\author{
Myung Il Kim and Sung Jo Kim \\ Dept. of Computer Science \& Engineering, Chung-Ang University, \\ 221 Huksuk-Dong, Dongjak-Ku, Seoul 156-756, South Korea \\ \{nicemi, sjkim\}@konan.cse.cau.ac.kr
}

\begin{abstract}
Multimedia applications are much more sensitive to the quality of service(QoS) than text-based ones due to their data continuity. In order to provide a fast moving mobile host $(\mathrm{MH})$ supporting multimedia applications with a consistent QoS, an efficient call admission mechanism is in need. This paper proposes the 2-level call admission control(2LCAC) scheme based on a call admission scheme using the priority to guarantee the consistent QoS for mobile multimedia applications. The $2 L C A C$ consists of the basic call admission and advanced call admission; the former determines call admission based on bandwidth available in each cell and the latter determines call admission by utilizing delay tolerance time $(D T T)$, priority queue(PQueue), and upgrade queue(UpQuеие) algorithms. In order to evaluate the performance of our scheme, we measure the metrics such as the blocking probability of new calls, dropping probability of handoff calls, and bandwidth utilization. The result shows that the performance of our scheme is superior to that of existing schemes such as complete sharing policy $(C S P)$, guard channel policy $(G C P)$ and adaptive guard channel policy $(A G C P)$.
\end{abstract}

\section{Introduction}

The provision of QoS in mobile multimedia networks becomes more complex due to user mobility. The problem becomes even more challenging as recent wireless networks are constructed based on small-size cells(microcells or picocells). Small-size cells increase the number of handoffs, which results in complex and rapid handling in call admission control $(C A C)$, thus increasing the handoff dropping rate.

It is important to reduce the dropping rate caused by lack of available bandwidth in the handoff target cell. If the cell's available bandwidth is not sufficient enough to satisfy a handoff call, this call has to compete with other calls to acquire the bandwidth. To solve this problem, several schemes have been proposed in literature for efficient channel assignment.

\footnotetext{
* This work was supported by grant No. R01-2001-000-00333-0 from the Korea Science \& Engineering Foundation.
} 
One well-known scheme is priority-based $C A C$ and channel assignment. From the user's point of view, dropping an ongoing call is more undesirable than blocking a new call[1],[2],[3]. As a result, many researches have been devoted to $C A C$ and channel assignment using prioritized handoff requests. Another well-known scheme is bandwidth reservation based $C A C$ which reserves bandwidth for handoff calls in all or some cells that $\mathrm{MH}$ might pass through, resulting in potentially lower bandwidth utilization and higher new call blocking probability.

In this paper, we propose the 2-level call admission control(2LCAC) scheme using the priority to guarantee the consistent QoS for mobile multimedia applications. A cell is assumed to be small and overlapped partially with its neighboring cells. $\mathrm{MH}$ calls are classified into new calls, hand-off calls, and QoS upgrading calls. The $2 L C A C$ consists of two stages: basic call admission control $(B C A C)$ and advanced call admission control $(A C A C)$. The former determines call admission based on available bandwidth in each cell and the latter determines call admission by utilizaing delay tolerance time $(D T T)$, priority queue(PQueue), and upgrade queue(UPQueue) algorithms according to the type of call blocked at $B C A C$.

We compare the performance of $2 L C A C$ scheme with that of the other $C A C$ schemes. Simulation results show that our scheme outperforms the others by satisfying the constraint on call blocking probability of new calls $(C B P)$, call dropping probability of handoff calls $(C D P)$ and bandwidth utilization.

This paper is organized as follows. We describe our $C A C$ scheme in Section 2. Section 3 shows simulation results and compares the performance of our scheme with that of the other schemes under various scenarios. Finally, Section 4 contains the concluding remarks.

\section{LCAC Scheme Based on Priority Queue}

Generally, it is assumed that a cell is hexagonal in mobile networks. However, in real world, a cell is close to a circle, and there are overlapped areas with neighboring cells as shown in Fig. 1. MHs residing in overlapped areas have higher possibility to move to another cell than MHs not residing in those areas.

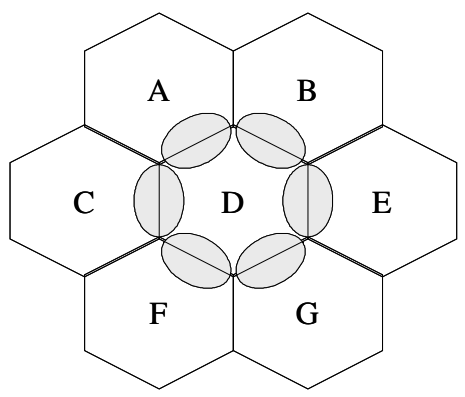

Fig. 1. Overlapped Cell Structure 


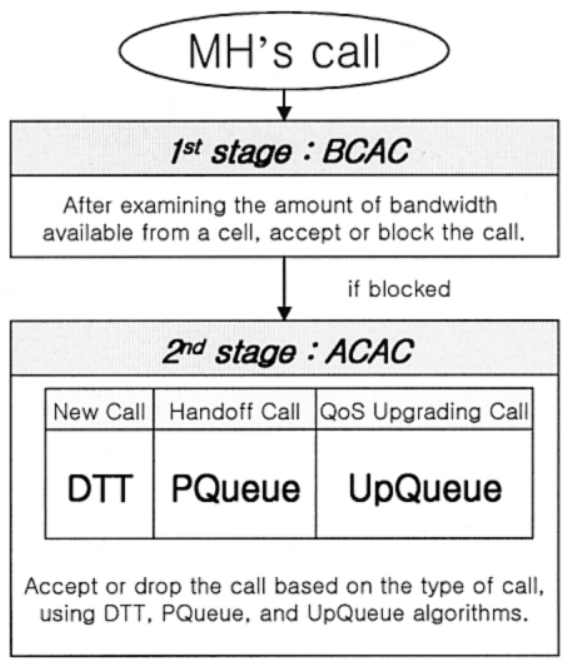

Fig. 2. $2 L C A C$ Procedure

As shown in Fig. 2, $2 L C A C$ consists of $B C A C$ and $A C A C$. At the $B C A C$ stage, each call(new, handoff and QoS upgrading) requests required bandwidth from its base station(BS). That is, $C A C$ is performed at the $B C A C$ stage when a call is requested for the first time from a specific cell. If available bandwidth of the BS satisfies each call's request, it assigns the bandwidth to the $\mathrm{MH}$; otherwise, it blocks the call and passes its control to the $A C A C$ stage.

Table 1. The Policies related to $B C A C$

\begin{tabular}{|c|c|c|c|}
\hline Call Type & Triggered Time & Conditions & Actions \\
\hline New & When $M H_{i}$ requests & $\begin{aligned} B_{j}^{\text {avail }} & \geq B_{i}^{\text {new }} \\
B_{j}^{\text {avail }} & <B^{\text {new }}{ }_{i}\end{aligned}$ & $\begin{array}{l}\text { Accept } \\
\text { Block and per- } \\
\text { form } A C A C \text { stage }\end{array}$ \\
\hline Handoff & $\begin{array}{l}\text { When } M H_{i} \text { enters } \\
\text { overlapped area }\end{array}$ & $\begin{array}{l}B_{j}^{\text {avail }} \geq B_{i}^{\text {handoff }} \\
B_{j}^{\text {avail }}<B_{i}^{\text {handoff }}\end{array}$ & $\begin{array}{l}\text { Accept } \\
\text { Block and per- } \\
\text { form } A C A C \text { stage }\end{array}$ \\
\hline $\begin{array}{l}\text { QoS } \\
\text { Upgrading }\end{array}$ & $\begin{array}{l}\text { When } B^{a v a i l} \text { is higher } \\
\text { than } T h_{u p}\end{array}$ & $\begin{array}{l}B_{j}^{\text {avail }} \geq T h_{\text {up }} \quad B_{j}^{\text {avail }} \geq B^{\text {up }}{ }_{i} \\
B^{\text {avail }}{ }_{j}<B^{\text {up }}{ }_{i} \\
B_{j}^{\text {avail }}<T h_{u p}\end{array}$ & $\begin{array}{l}\text { Accept } \\
\text { Block and per- } \\
\text { form } A C A C \text { stage } \\
\text { Block and per- } \\
\text { form } A C A C \text { stage }\end{array}$ \\
\hline
\end{tabular}

\subsection{Basic Call Admission Control (BCAC)}

Table 1 shows the policies related to $B C A C$, which are chosen by the type of calls. $B^{\text {new }}{ }_{i}$ and $B^{\text {handoff }}{ }_{i}$ represent $M H_{i}$ 's required bandwidth of new call and handoff call, re- 
spectively. Whereas $B^{u p}{ }_{i}$ represents required bandwidth of QoS upgrading call, $B^{\text {avail }}$ represents the amount of bandwidth available in cell $j . B_{j}^{\text {avail }}$ can be calculated by Eq. (1):

$$
B_{j}^{\text {avail }}=B_{j}^{\text {total }}-\left(\sum_{i=0}^{n} B^{\text {handoff }}+\sum_{k=0}^{m} B_{k}^{\text {new }}\right)
$$

where $B^{\text {total }}{ }_{j}$ is the total amount of bandwidth provided by cell $j$ and $n$ and $m$ are the number of handoff calls and new calls currently residing in cell $j$, respectively.

In case of a new call, the $C A C$ is triggered when $\mathrm{MH}$ requests the call admission. If $B^{\text {avail }}{ }_{j}$ is larger than $B^{\text {new }}$, the call is admitted; otherwise, blocked. In case of a handoff call, the $C A C$ is triggered when the MH enters an overlapped area. If the $B^{\text {avail }}{ }_{j}$ is larger than $B_{i}^{\text {handoff }}$, the call is admitted; otherwise, blocked. In order to upgrade the current QoS level of an ongoing call, additional bandwidth can be requested by triggering $C A C$. If $B^{\text {vvail }}{ }_{j}$ is larger than $T h_{u p}$, the call is accepted, where $T h_{u p}$ is the threshold used for determining the acceptance of QoS upgrading call; otherwise, blocked. The control of blocked new, handoff and QoS upgrading call will be treated at the ACAC stage.

\subsection{Advanced Call Admission Control (ACAC)}

\section{Priority Queue and QoS Upgrading Queue}

In $A C A C$, each cell requires space to store information about $\mathrm{MH}$ : priority queue $(P Q)$ and QoS upgrading queue $(U p Q) . P Q$ stores information on an MH such as ID and the priority that has been blocked as a result of $B C A C$ for its handoff. For example, when $\mathrm{MH}_{1}$ and $\mathrm{MH}_{2}$ move towards the overlapping area with cell $D$ from cell $C$ and $E$, respectively, as shown in Fig. 3, BCAC is triggered. If both MHs are blocked, the priorities of $\mathrm{MH}_{1}$ and $\mathrm{MH}_{2}$ are calculated by cell $D$ 's BS and the information on those blocked MHs are inserted into $P Q$. Furthermore, if an $\mathrm{MH}_{3}$ residing in cell $D$ moves towards cell $B$ through the overlapped area between the two cells and is blocked as a result of $B C A C$, the priority of $\mathrm{MH}_{3}$ is inserted into cell $B$ 's $P Q$.

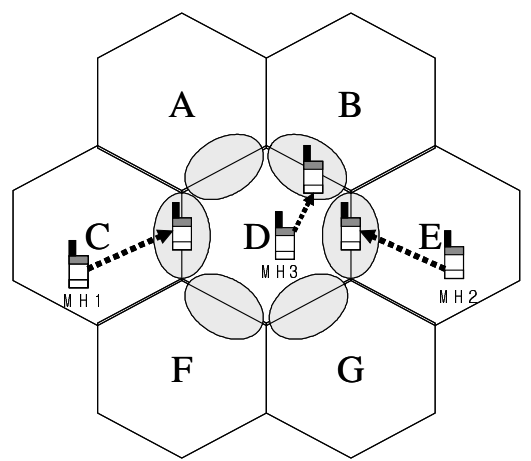

Fig. 3. A Snapshot of Cell Status 
The call priority of $\mathrm{MH}$ is calculated using Eq. (2):

$$
\text { Call priority }=w_{1} D P+w_{2} T P+w_{3} V P
$$

where $D P, T P$ and $V P$ are the distance priority, traffic priority and velocity priority, respectively, and $w_{1}, w_{2}$ and $w_{3}$ are weight factors. The rationale behind this equation is that an $\mathrm{MH}$ has the highest priority as it gets closer to the target cell $(D P)$ at a high mobility speed $(V P)$ and require the service with high $T P$ like multimedia services.

QoS upgrading calls that have been blocked as a result of $B C A C$ are inserted into $U p Q$. When the amount of bandwidth available in a cell is greater than $T h_{u p}$, the bandwidth is allocated to the calls in $U p Q$ in the FIFO order.

\section{DTT, PQueue and UPQueue Algorithm}

In $A C A C$, different $C A C$ algorithms are adopted according to the type of each call; new, handoff and QoS upgrading calls are tested using DTT, PQueue and UPQueue algorithm, respectively. In case of a new call, it requests a call admission to BS periodically until DTT expires, where DTT is set when the $\mathrm{MH}$ is powered on. If available bandwidth of BS is not enough to satisfy the new call's request after DTT, it is dropped.

In case of handoff calls, call classification and bandwidth borrowing algorithms are invoked. Call classification algorithm as below is applied to MHs that have just entered the overlapped area. It classifies handoff calls into two classes: high and low. Class High contains handoff calls that have relatively higher priority than the others. Once being classified into this class, the calls will not be degraded into class low and bandwidth assignment to them will be also guaranteed. On the other hand, class low contains handoff calls that have relatively lower priority than the others. They have high chances of being dropped during $A C A C$ and bandwidth assignment to them will not be guaranteed.

Pseudo Code for Call Classification Algorithm

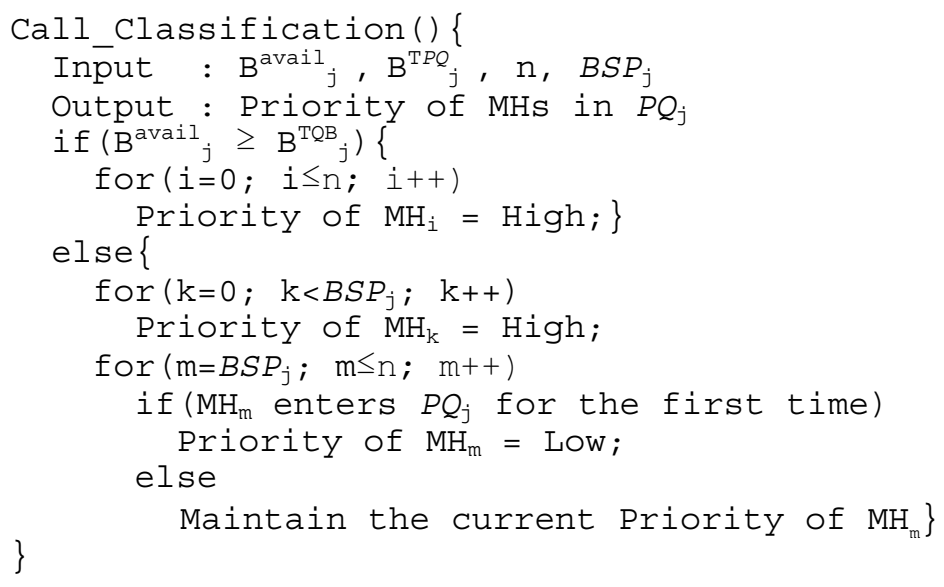


In the pseudo code above, $B^{T P Q}$ (Total Bandwidth of $P Q$ ) is the sum of bandwidth requested by all the MHs that reside in cell $j$ 's $P Q$. Bandwidth satisfaction point $\left(B S P_{j}\right)$ points out the right most $\mathrm{MH}$ in $P Q$ of which bandwidth request can not be satisfied from cell $j$ 's available bandwidth. To be specific, if $B_{j}^{\text {avail }}$ is equal to or higher than $B^{T P Q}$, the priorities of all the MHs residing in $P Q$ become high. However, if $B_{j}^{\text {avail }}$ is lower than $B_{j}^{T P Q}$, only the priorities of MHs that are at the left-hand side of $B S P_{j}$ become high and the others become low. The MHs that are at the right-hand side of $B S P$ and have a high priority maintain its current priority. MHs residing in $P Q$ are sorted based on their priorities.

When the priority of $M H_{i}$ becomes high during its stay in the overlapped area, the required bandwidth is assigned to it if the amount of bandwidth available in the target cell is sufficient enough, or bandwidth borrowing algorithm[1],[3],[4] is triggered to assign the amount of bandwidth requested by $M H_{i}$. On the other hand, the algorithm is not triggered immediately if the priority of $M H_{i}$ belongs to class low, and the triggering of the algorithm is delayed until its priority is upgraded to class high. A handoff call of $M H_{i}$ is dropped, if the priority of $M H_{i}$ cannot be upgraded to class high until it leaves the overlapped area.

In case of QoS upgrading call, an $\mathrm{MH}$ is stored into the QoS upgrading queue in order to upgrade the QoS level. This happens either when the MH wants to upgrade its service quality or to reclaim as much bandwidth as it has lent to the other MH. Only when the amount of bandwidth available in BS is above the threshold, the required bandwidth is assigned. Just because there is sufficient bandwidth in a cell, the QoS will not be upgraded immediately by UPQuеиe algorithm. To be specific, if the available bandwidth is below the threshold, $M H_{i}$ is stored in the QoS upgrading queue. Next, only when the available bandwidth is above the specific threshold, the QoS of $\mathrm{MH}_{i}$ is upgraded in the FIFO order. Note that the available bandwidth below the threshold is assigned to new calls and handoff calls, because the QoS upgrade calls have the lower priority than both new calls and handoff calls.

\section{Performance Evaluation}

In this section, we compare the performance of proposed scheme with that of other schemes such as CSP, GCP, and AGCP. In CSP [5], new and handoff calls are not distinguished from each other and are admitted as long as available bandwidth of a cell can provide the required bandwidth to the $\mathrm{MH}$. In other words, the same priority is given to both the new and handoff calls. This scheme has the low $C B P$, but high $C D P$.

$G C P$ [5],[6],[7] reserves a fixed amount of bandwidth for multimedia handoff calls. This scheme reserves as much bandwidth as required by an $\mathrm{MH}$ requesting multimedia services and having high probability of entering a certain cell. The bandwidth is assigned later when the MH actually enters the cell. This scheme has an advantage of having the low $C D P$ for multimedia handoff calls by assigning reserved bandwidth to them. However, since the available bandwidth is reserved for multimedia calls, new 
and non-multimedia handoff calls have high $C B P$ and $C D P$. Moreover, the reserved bandwidth may turn out not to be used, thus resulting in low bandwidth utilization.

$A G C P$ [2],[8],[9] is a policy that adaptively reserves a portion of bandwidth for multimedia handoff calls depending on network condition. If the $C D P$ of a cell is above the specific threshold (e.g., 0.05), the amount of reserved bandwidth is increased. On the other hand, if the $C B P$ is below a certain threshold, a certain amount of reserved bandwidth is decreased only when the utilization of reserved bandwidth is below specific threshold (e.g. 0.5). This scheme has the lower CDP than GCP. When the number of new calls increases dramatically, however, the $C D P$ is increased as well.

Table 2. Traffic Classes Used in the Simulation

\begin{tabular}{llll}
\hline $\begin{array}{l}\text { Traffic } \\
\text { Class }\end{array}$ & $\begin{array}{l}\text { Bandwidth } \\
\text { Requirement }\end{array}$ & $\begin{array}{l}\text { Call Duration } \\
\text { (minutes) }\end{array}$ & Typical Service \\
\hline \multirow{3}{*}{ Class I } & $30 \mathrm{Kbps}$ & $1 \sim 10$ & Voice Service \\
& $256 \mathrm{Kbps}$ & $1 \sim 30$ & Video-Phone \\
& $1 \sim 6 \mathrm{Mbps}$ & $5 \sim 300$ & Video on Demand \\
\hline \multirow{2}{*}{ Class II } & $32 \mathrm{Kbps}$ & $0.2 \sim 2$ & E-mail, Paging \\
& $64 \sim 512 \mathrm{Kbps}$ & $0.5 \sim 600$ & Remote Login \& \\
& $1 \sim 10 \mathrm{Mbps}$ & $0.5 \sim 20$ & Fata on Demand \\
\hline
\end{tabular}

In this simulation, we suppose that the mobile multimedia network is comprised of 100 hexagonal cells, each of which diameter is $1 \mathrm{~km}$. We also suppose that each cell provides the bandwidth of $30 \mathrm{Mbps}$ and communicates with its six neighboring cells. The client moves at a speed from 1 to $100 \mathrm{Km} / \mathrm{h}$. New call reqeusts are generated according to a Poisson distribution with the arrival rate $\lambda$ (calls/second) in each cell. A new call is assumed to be requested at any place in the cell with an equal probability. Table 2 shows six typical traffic classes used in the simulation[8].

Performance metrics considered in the simulation are $C B P, C D P$ and bandwidth utilization. The metrics are measured with respect to $C S P, G C P$, and $A G C P$ as the arrival rate of new calls vary.

We first attempt to find out the most reasonable rate of class I calls by performing our simulation under the situation as follows; the arrival rate of new calls is fixed at $0.1(\mathrm{MH} / \mathrm{sec})$ and the proportion of class I calls is incremented by $10 \%$ from $10 \%$ to $90 \%$. We find that the $C B P, C D P$, and bandwidth utilization showed the best performance when the proportion of class I call is $50 \%$. We then perform our simulation under the following situation; the proportion of class I calls is fixed at $50 \%$ and the arrival rate of new calls is incremented by $0.01(\mathrm{MH} / \mathrm{sec})$ from 0.01 to 0.1 .

Fig. 4 shows the $C B P$ for each of four schemes as the arrival rate of new calls increases from 0.01 to 0.1 . According to the figure, $2 L C A C$ has the lowest $C B P$ followed by $C S P$. It also shows that $A G C P$ has the highest $C B P$. This is because $2 L C A C$ allows an $\mathrm{MH}$ to request the acceptance of its blocked new call repeatedly at the 
$B C A C$ stage until $D T T$ expires without human-user's intervention. The $C B P$ of $A G C P$ and $G C P$ are lower than that of $C S P$. The reason for that is that $A G C P$ and $G C P$ assign higher priority to handoff calls than new calls and reserve some bandwidth for handoff calls. As a result, as the amount of bandwidth assigned to new calls decreases, the $C B P$ s of $A G C P$ and $G C P$ are higher than those of $2 L C A C$ and $C S P$. On the other hand, the CSP treats new and handoff calls equally and does not reserve bandwidth for handoff calls.

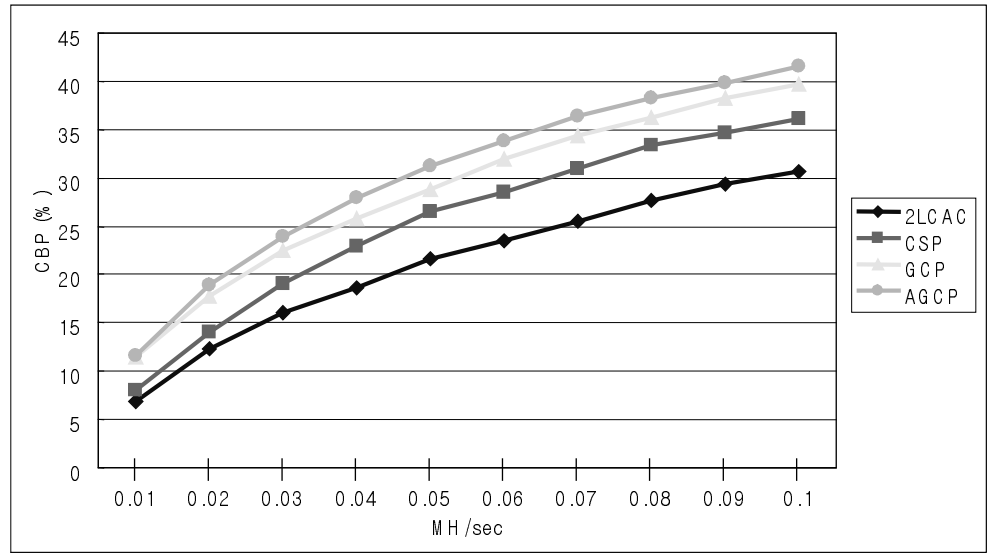

Fig. 4. Blocking Probability of New Calls

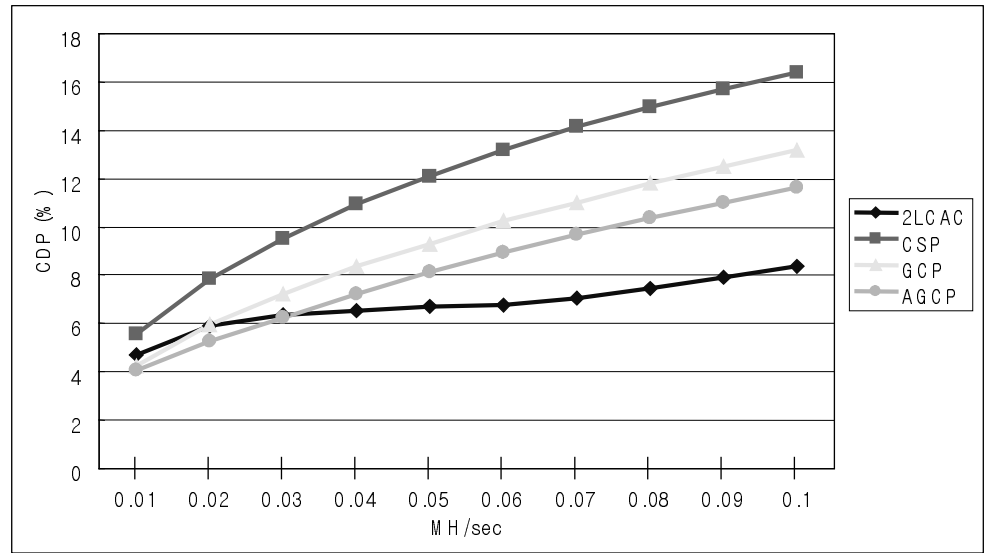

Fig. 5. Dropping Probability of Handoff Calls

Fig. 5 shows the $C D P$ of each scheme as the arrival rate of new calls increases from 0.01 to 0.1 . $2 L C A C$ has the lowest $C D P$, followed by $A G C P, G C P$, and $C S P$. This is because $2 L C A C$ gives a priority to both class I and class II handoff calls efficiently and fairly. Fair bandwidth assignment is guaranteed by $2 L C A C$ based on the type of calls, the speed of MH, and MH's distance from the target cell. Even when there is not 
enough bandwidth available in a cell, handoff call admission is guaranteed for handoff calls with high priority by reclaiming bandwidth at the $A C A C$ stage. If there are many new call requests, the $C S P$ has the highest $C D P$ because it does not assign higher priority to handoff calls than new calls. In case of $A G C P$ and $G C P$, both have the high $C D P$ because the reserved bandwidth might not be used at all and be eventually wasted.

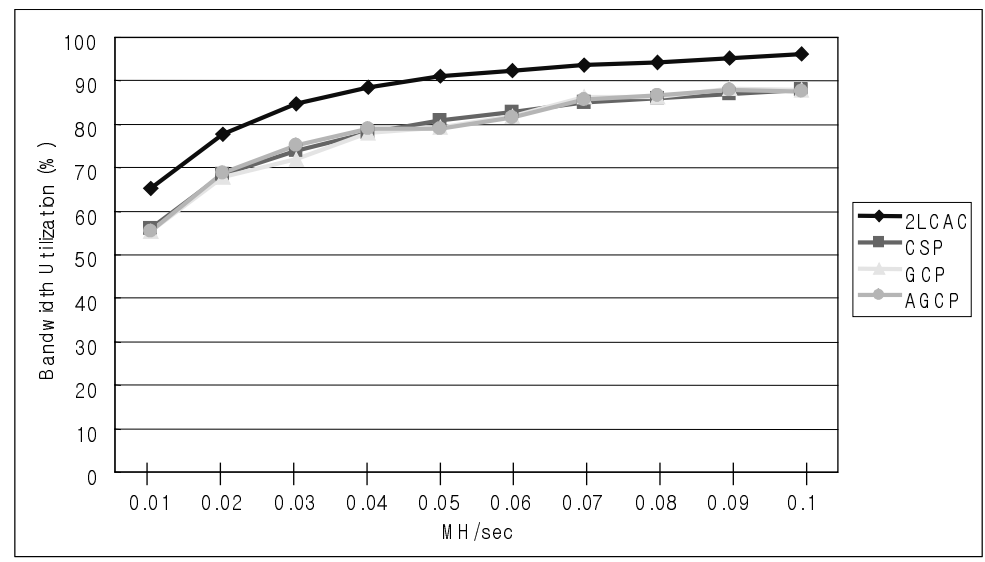

Fig. 6. Bandwidth Utilization

Fig. 6 shows the bandwidth utilization of each scheme. $2 L C A C$ has the highest utilization followed by $C S P, G C P$, and $A G C P$. This is because $2 L C A C$ uses available bandwidth of a cell more efficiently than other schemes. When there is available bandwidth in a cell, it is assigned to QoS upgrade calls. Since QoS upgrade calls of an $\mathrm{MH}$ reclaim bandwidth which has been previously lent to handoff calls with high priority, $2 L C A C$ manage to utilize the bandwidth efficiently. On the other hand, $A G C P$ and $G C P$ have low bandwidth utilization, because reserved bandwidth can be unused and be wasted.

\section{Conclusion}

For efficient and fair $C A C$, we proposed $2 L C A C$ scheme using priority queue. We classified MH calls into three types: new, handoff, and QoS upgrade call. The priority of each type is assigned in the order of handoff, new, and QoS upgrade call, and each type uses the different call admission policy. $2 L C A C$ consists of two stages: BCAC and $A C A C$. At the former stage, calls from an $\mathrm{MH}$ are admitted without distinguishing new and handoff calls just as the CSP, when the amount of bandwidth available in a cell satisfies the required bandwidth of the MH. In case of QoS upgrading calls, they are accepted if the available bandwidth is above the specific threshold; otherwise, the calls are blocked and $A C A C$ is triggered. The latter stage tests whether the calls 
blocked at the former stage can be accepted or not using DTT, PQuеиe, and UPQueue algorithms depending on the type of calls.

In order to evaluate the performance of our scheme, we measured the metrics such as $C B P, C D P$, and bandwidth utilization. We then compared the performance of $2 L C A C$ with that of three different schemes: CSP, GCP, and AGCP. Because CSP does not distinguish new calls from handoff calls, it has the low $C B P$ and high $C D P$. Since $G C P$ reserves a fixed amount of bandwidth, it has the low $C D P$, but the highest $C B P$. This scheme has the lowest bandwidth utilization because it cannot adaptively readjust the amount of bandwidth for handoff calls. The performance of $A G C P$ is better than that of other schemes, but still worse than that of $2 L C A C$. To be specific, $2 L C A C$ lowers its $C B P$ and $C D P$ by $6 \sim 11 \%$ and $5 \sim 8 \%$, respectively, compared to $C S P, G C P$ and $A G C P$. The bandwidth utilization of $2 L C A C$ is also better than that of other schemes by 7 8\%. All in all, $2 L C A C$ utilizes the bandwidth of BS more efficiently than other conventional schemes.

\section{References}

1. W. Zhuang, B. Bensaou, and K. Chua, "Adaptive Quality of Service Handoff Priority Scheme for Mobile Multimedia Networks," IEEE Transactions on Vehicular Technology, Vol.49, No.2, pp.494-505, 2000.

2. C. H. Choi, M. I. Kim and S. J. Kim, "Call Admission Control Using Moving Pattern of Mobile Users for Mobile Multimedia Networks," IEEE Conference on Local Computer Networks, pp.431-440, 2002.

3. M. Ei-Kaid, S. Olariu and H. Abdel-Wahab, "Rate-Based Borrowing Scheme for QoS Provisioning in Multimedia Wireless Networks," IEEE Transaction on Parallel and Distributed Systems, Vol. 13, No. 2, pp.156-166, 2002.

4. A. Xhafa and O. K. Tonguz, "Dynamic Priority Queuing, Channel Borrowing, and First In First Out Handoff Schemes: A Performance Comparison," IEEE Vehicular Technology Conference Spring, Vol.2, pp.951-955, 2001.

5. B. Gavish and S. Sridhar, "Threshold Priority Policy for Channel Assignment in Cellular Networks," IEEE Transactions on Computers, Vol.46, No.3, 1997.

6. J. Lee, et al., "An Adaptive Resource Allocation Mechanism including Fast and Reliable Handoff in IP-based 3G Wireless Networks," IEEE Personal Communications, Vol.7, No.6, pp.42-47, 2000.

7. E. El-Alfy, Y. Yao, and H. Heffes, "A Learning Approach for Call Admission Control with Prioritized Handoff in Mobile Multimedia Networks," IEEE Vehicular Technology Conference, Vol.2, pp.972-976, 2001.

8. C. Oliveria, J. Kim and T. Suda, "An Adaptive Bandwidth Reservation Scheme for High Speed Multimedia Wireless Networks," IEEE JSAC, Vol.16, No.6, pp.858-874, 1998.

9. S. Choi and K. Shin, "Predictive and Adaptive Bandwidth Reservation for Hand-offs in QoS-Sensitive Cellular Networks," Proceeding ACM SIGCOMM'98, pp.155-166, 1998. 